\title{
Reconditioning acidic and artificially aged cellulose with alkaline nanoparticles: an NMR diffusometry study
}

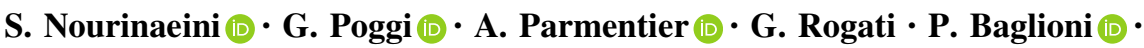 \\ F. De Luca
}

Received: 15 November 2019/Accepted: 17 June 2020/Published online: 26 June 2020

(C) Springer Nature B.V. 2020

\begin{abstract}
The prominent degradation mechanism of cellulose is the acid-catalyzed hydrolysis of glycosidic bonds, which results in the decrease of the degree of polymerization (DP) and, macroscopically, in the dramatic decay of the mechanical resistance of cellulose-based materials. Alkaline nanoparticles in organic solvents have been recently proposed for the deacidification of cellulose-based artworks. Their effectiveness has been demonstrated in previous studies, by $\mathrm{pH}$ and DP measurements, colorimetric and thermal analyses. Herein, the changes in the
\end{abstract}

S. Nourinaeini · G. Rogati · F. De Luca

Department of Physics, Sapienza University of Rome, P.le

A. Moro 2, 00185 Rome, Italy

e-mail: solmaz.nourinaeini@uniroma1.it

G. Rogati

e-mail: giovanna.rogati@roma1.infn.it

F. De Luca

e-mail: francesco.deluca@roma1.infn.it

G. Poggi $(\bowtie) \cdot$ P. Baglioni

Department of Chemistry and CSGI, University of

Florence, Via della Lastruccia 3,

50019 Sesto Fiorentino, Florence, Italy

e-mail: poggi@csgi.unifi.it

P. Baglioni

e-mail: baglioni@csgi.unifi.it

A. Parmentier

INFN - Division of Rome Tor Vergata, Via della Ricerca

Scientifica 1, 00133 Rome, Italy

e-mail: alexandra.parmentier@roma2.infn.it cellulosic network following an acidification bath and a consequent deacidification treatment using $\mathrm{Ca}(\mathrm{OH})_{2}$ nanoparticles, have been investigated by NMR selfdiffusion dynamics of water and related to the changes of samples' DPs. The deacidification treatment modifies intra- and inter-chain interactions, leading to a buffered cellulose network configuration similar to that characterizing the untreated reference sample in terms of diffusive parameters and confining environment. Such results are plausibly due to a rearrangement in connectivity of the cellulosic network, even though with a different physical fingerprint with respect to the reference sample. The analysis of tortuosity of the cellulosic network in acidic and deacidified samples confirms this conclusion, further corroborating the idea that calcium hydroxide nanoparticles are an effective tool to hamper the degradation of cellulose induced by acids and aging in strong environmental conditions, even from the standpoint of cellulose network arrangement.

Keywords Cellulose - NMR diffusometry · Deacidification - Calcium hydroxide nanoparticles . Hydrolysis

\section{Introduction}

Cellulose is a linear polymer found in plants, which plays a fundamental role in the mechanical properties 
of vegetal tissues. Cellulose in nature forms chain aggregates, i.e., hierarchical structures that range from elementary fibrils, having a transverse extension of a few nm, to microfibrils, which are aggregates of tens of elementary fibrils (Fernandes et al. 2011; Ding et al. 2014; Paajanen et al. 2019). Microfibrils are arranged in macrofibrils, or fibers, whose transverse size may be tens of $\mu \mathrm{m}$ (Zhao et al. 2007; Donaldson 2007; Paajanen et al. 2019). These supramolecular structures are formed through a coalescence-like mechanism, which favors the formation of hydrogen bonds between $\mathrm{OH}$ groups of different chains, and makes Van der Waals interactions mostly effective for fiber formation (Niskanen 1998).

In elementary fibrils, crystalline and amorphous regions, having a different chain packing, can be found (Fengel and Wegener 1984; Niskanen 1998). Chains in crystalline regions are packed in crystalline structures and, therefore, they are impenetrable to water and highly resistant to chemical agents. Conversely, amorphous domains (ADs) show little or no chain order. The AD regions behave as hydrophilic sites, where water, as well as chemical agents, may interact with single cellulose chains (Topgaard and Söderman 2001; Kulasinski et al. 2015); ADs are, therefore, more prone to degradation (Stephens et al. 2008).

It has been recently proposed that water in cellulose is arranged in two phases characterized by different confining environments, both involving ADs (Conti et al. 2014, 2017). A phase characterized by more mobile water (phase-1) is assigned to ADs located in elementary fibrils at fiber surfaces, while less mobile water (phase-2) is confined in microfibril ADs located at fiber cores (Müller et al. 2000; Schuster et al. 2003; Zhao et al. 2007; Conti et al. 2017). This model accounts well for experimental data, and it fully agrees with the widespread phenomenological characterization of water in cellulose structures reported in the literature.

Most degradation mechanisms of cellulose ADs take place in an acidic environment (Fengel and Wegener 1984). The most important is, indeed, the acid-catalyzed hydrolysis of the $\beta$-1,4-glycosidic bonds of chains, which results in the decrease of the degree of polymerization of cellulose and, macroscopically, in the dramatic decay of the mechanical resistance of cellulose-based materials (Zervos and Moropoulou 2005; Arroyo et al. 2017). Since several materials involved in the manufacturing process of cellulosic objects are acidic, or may develop acidic compounds upon aging, cellulose-based artworks are likely to experience an irreversible decay, or are already in need of urgent maintenance (Dobrodskaya et al. 2004; Strlič and Kolar 2005; Wouters 2008; Yanjuan et al. 2013; Afsharpour and Hadadi 2014). Deacidification treatments can be used to hinder the acid-catalyzed degradation of cellulose-based artifacts (Baty et al. 2010; Zervos and Alexopoulou 2015). Calcium or magnesium hydroxide nanoparticles stably dispersed in poorly polar, or non-polar, solvents have been recently proposed for the deacidification of cellulose-based artworks. Alkaline nanoparticles have been successfully applied on wood (Giorgi et al. 2005b; Poggi et al. 2016b), paper and canvas (Giorgi et al. 2002, 2005a; Poggi et al. 2010, 2014, 2017), and underwent extensive assessment with positive results (Sequeira et al. 2006; Stefanis and Panayiotou 2007, 2008; Bastone et al. 2017). More recently, alkaline nanoparticles have been used in association with strengthening materials, to achieve the mechanical stabilization of acidic artworks (Poggi et al. 2016a; Palladino et al. 2020; Xu et al. 2020). The effectiveness of this method for the protection of cellulose-based artworks from acidic degradation has been demonstrated by $\mathrm{pH}$ and $\mathrm{DP}$ measurements, colorimetric and thermal analyses. On the other hand, to the best of our knowledge, the effects of alkaline nanoparticles, and, more in general, of a deacidification treatment on the cellulosic network arrangement have never been studied.

To this end, untreated, acidic and deacidified samples underwent a two-week aging at high temperature and relative humidity, which is commonly used to induce the depolymerization of cellulose. Since acidification and artificial aging trigger various processes that influence intra- and inter-chain interactions (Kato and Cameron 1999; Zervos 2007), the arrangement of cellulose might result rather modified. The resulting modifications in samples may allow to establish whether alkaline nanoparticles work as a successful conservation method even from the standpoint of cellulose arrangement.

The changes in cellulose arrangement are herein indirectly investigated by NMR self-diffusion dynamics of water trapped in the cellulose network and related to the degree of polymerization of the investigated samples. This case can be embedded in the general context of self-diffusion of water in a multi- 
phase system, which is effectively described by the Kärger model (Kärger et al. 1988) that provides information about confining sizes and net connectivity when suitable NMR measurement procedures and data processing are implemented (Conti et al. 2014).

\section{Materials and methods}

\section{Sample preparation}

Whatman filter paper (grade 5, 125-mm diameter) composed of raw cotton fibers (minimum $\alpha$-cellulose content: 98\%) was used for the preparation of the samples. Acidic (A) samples were prepared by immersion of filter paper disks in a $\mathrm{H}_{2} \mathrm{SO}_{4}$ solution $(\mathrm{pH}=2.5)$ for $240 \mathrm{~s}$. Prior to measurements, samples were left under ambient conditions for 2 weeks.

Untreated filter paper disks were used as a reference. Since capillarity and pore size can be altered by immersion in water (Topgaard and Söderman 2001), reference systems were immersed in distilled water for $240 \mathrm{~s}$ to prevent the effects from mere soaking (Conti et al. 2014). These samples are labeled as R.

Deacidified samples, labeled as $\mathrm{D}$, were prepared as follows: filter paper disks underwent the same acidification process as A samples; after 2 weeks, each disk was treated with $3 \mathrm{ml}$ of calcium hydroxide nanoparticle dispersion in ethanol $(1.5 \mathrm{~g} / \mathrm{L})$. Calcium hydroxide nanoparticles, synthesized via a solvothermal process starting from metal calcium and ethanol, are crystalline hexagonal platelets, having a thickness of about 20-30 nm and an average diameter (obtained by dynamic light scattering measurements) of about $200 \mathrm{~nm}$ (Poggi et al. 2014, 2016a). The nanoparticle dispersion was applied on both disk sides using a micropipette, pouring the liquid on the sample to homogeneously wet the surface. Samples were then left under ambient condition for 2 weeks.

To trigger the degradation of cellulose, samples were artificially aged under strong hydrothermal conditions. Samples were placed in a vessel together with a sodium chloride saturated solution, which was used to maintain the relative humidity at about $75 \%$. The vessel was then sealed and placed in an oven at $80{ }^{\circ} \mathrm{C}$. Samples were aged for 2 weeks; 1 -week aged samples are labelled as R1, A1, D1, and 2-weeks aged samples as R2, A2 and D2.
Sample characterization

\section{pH and degree of polymerization (DP) measurements}

To perform $\mathrm{pH}$ measurements, $125 \mathrm{mg}$ of sample was weighed, cut into small pieces (about $9 \mathrm{~mm}^{2}$ ), and placed inside screw-top vials. $9 \mathrm{ml}$ of distilled water was added to each vial, immediately sealed to avoid the solubilization of $\mathrm{CO}_{2}$ from air into the extracting water. Vials were kept under stirring for one hour before measuring the $\mathrm{pH}$ of the extraction using a digital pH-meter (CrisonBasic 20, equipped with a combined electrode, model 52-21). Three measurements were performed on each sample. The associated error is \pm 0.2 .

The degree of polymerization of cellulose in samples was obtained by viscosimetric measurements (UNI 8282 1994). Data are presented in terms of scissions per initial cellulose chain $\left(S^{*}\right)$, calculated by means of the following equation (Dupont and Mortha 2004; Calvini et al. 2008):

$S^{*}=\frac{D P_{V_{0}}}{D P_{V_{t}}}-1$

where $D P_{V_{0}}$ is the degree of polymerization at time zero, and $D P_{V_{t}}$ is the degree of polymerization at any time $t$. An accurate calculation of the number of scissions cannot be obtained from $D P_{V}$ due to the fact that polydispersity cannot be assessed by viscosimetric measurements. Nevertheless, in the present work, the comparison of $S^{*}$ values calculated from $D P_{V}$ can be considered fully acceptable, because the experimental data refer to homologous series of samples. The error associated to these measurements is \pm 25 .

$\mathrm{PH}$ and $D P_{V}$ values were monitored before, during and after the aging, and are reported in Table 1.

\section{Diffusion measurements}

Measurements of the self-diffusion coefficient $D$ have been performed by means of a Bruker Avance $300 \mathrm{MHz}$ spectrometer equipped with a gradient unit that generates a maximum gradient intensity of about $1200 \mathrm{G} / \mathrm{cm}$. A pulsed field gradient spin-echo (PFGSTE) sequence was implemented (Callaghan 2011). In this sequence, two encoding/refocusing magneticfield-gradient pulses $G$ of $\delta \cong 1.4 \mathrm{~ms}$ duration are applied within three $90^{\circ}$ hard radio-frequency (rf) pulses. The first two rf pulses are separated by a time 
Table $1 D P_{V}, S^{*}, \mathrm{pH}$, apparent diffusion coefficient $\left(\mathrm{D}_{1}\right)$, confining size $\left(\mathrm{d}_{1}\right)$ and relative tortuosity $(\mathrm{T})$ of the analyzed paper samples

\begin{tabular}{|c|c|c|c|c|c|c|}
\hline Sample name & $D P_{V}$ & $S^{*}$ & $\mathrm{pH}$ & $\mathrm{D}_{1}\left[10^{-10} \mathrm{~m}^{2} / \mathrm{s}\right]$ & $\mathrm{d}_{1}[\mu \mathrm{m}]$ & $\mathrm{T}$ \\
\hline $\mathrm{R}$ & 920 & - & 6.7 & 0.12 & 2.5 & - \\
\hline R1 & 683 & $0.35 \pm 0.02$ & 6.9 & 0.10 & 2.2 & - \\
\hline R2 & 555 & $0.66 \pm 0.05$ & 6.9 & 0.08 & 2 & - \\
\hline A & 551 & $0.67 \pm 0.05$ & 4.8 & 0.21 & 3 & 0.6 \\
\hline A1 & 349 & $1.64 \pm 0.16$ & 5.4 & 0.08 & 2.2 & 0.6 \\
\hline $\mathrm{A} 2$ & 345 & $1.67 \pm 0.17$ & 5.3 & 0.06 & 1.8 & 0.9 \\
\hline D & 537 & $0.71 \pm 0.05$ & 9.7 & 0.12 & 2.3 & 0.5 \\
\hline D1 & 496 & $0.85 \pm 0.07$ & 9.2 & 0.12 & 2.2 & 0.3 \\
\hline D2 & 504 & $0.82 \pm 0.06$ & 8.9 & 0.08 & 2 & 0.9 \\
\hline
\end{tabular}

The errors related to $D P_{V}$ and $\mathrm{pH}$ are \pm 25 and \pm 0.2 respectively, those of $\mathrm{D}_{1}$ and $\mathrm{d}_{1}$ are indicated in the corresponding graphs, and the error related to $\mathrm{T}$ is within $10 \%$

interval $\tau_{0} \cong 2.0 \mathrm{~ms}$, while the second field gradient has a delay $\Delta$, the diffusion time, with respect to the first. A set of different $\Delta$ values was tested, up to a maximum value of $60 \mathrm{~ms}$. For each of the 20 gradient steps used (with gradient intensity spanning the range from zero to $1050 \mathrm{G} / \mathrm{cm}$ ), $32 \mathrm{NMR}$ scans were performed to improve the signal-to-noise ratio. The relaxation recycle delay was fixed at $3 \mathrm{~s}$. Hydrated paper samples were cut into strips of about $2.5 \times 20 \mathrm{~mm}^{2}$, sealed in a plastic film to avoid drying, and placed into the NMR tube. Measurements were performed at $22.0{ }^{\circ} \mathrm{C}\left( \pm 0.1{ }^{\circ} \mathrm{C}\right)$.

Under the narrow gradient-pulse approximation (Callaghan 2011), the echo amplitude $E(q, \Delta)$ is related to $D$ through the relation:

$E(q, \Delta) \cong E(0, \Delta) \exp \left[-q^{2} D \Delta\right]$

where $q=\gamma \delta G$ is the dynamic wave vector and $\gamma$ is the gyromagnetic ratio.

To prevent relaxational effects from spoiling the NMR signal, the safety conditions $\tau_{0} \ll T_{2 S}$ and $\Delta \ll T_{1 \mathrm{~S}}$ were established, where $T_{2 \mathrm{~S}}$ and $T_{1 \mathrm{~S}}$ are the shortest transverse $\left(T_{2}\right)$ and longitudinal $\left(T_{1}\right)$ NMR relaxation times found over the entire set of samples. Before measurements, samples were kept at $22 \pm 1{ }^{\circ} \mathrm{C}$ for $24 \mathrm{~h}$ in a $100 \%$ relative humidity environment.

\section{Results and discussion}

The Kärger model well describes the molecular diffusion-dependent NMR signal over two exchanging domains or phases (Kärger et al. 1988). Specifically, the model describes the PFG-STE signal as the sum of two simple spin-echo signals $E(q, \Delta)$ (see Eq. (2)), provided that the average residence times of molecules in their own domains are significantly larger than the diffusion time $\Delta$ (Conti et al. 2017). Figure 1 reports the fast and slow components of the PFG-STE diffusion coefficients measured in R, A and D samples before the artificial aging.

Figure 1 shows the presence of a clear dispersion on both diffusion components, which is a mark of restricted-diffusion dynamics. It is worth noting that the phase- 2 component shows little variability, being related to less mobile water confined in microfibril ADs located at fiber cores (Müller et al. 2000; Schuster et al. 2003; Zhao et al. 2007; Conti et al. 2017), and therefore it will not be further taken into account. On the other hand, the phase-1 component is well fitted by the function $D(\Delta)=D(0) \exp (-\Delta / \tau)+D(\infty)$. As can be seen in Fig. 1, the diffusion coefficient at high diffusion times is not close to zero, i.e., $D(\infty) \neq 0$, which confirms that the cellulose network of ADs at fiber surfaces behaves as an interconnected pore network (Callaghan 2011). However, the use of the mean square displacement (MSD) has been recently proven convenient to gather more information about the diffusion (Conti et al. 2017). MSD can be 

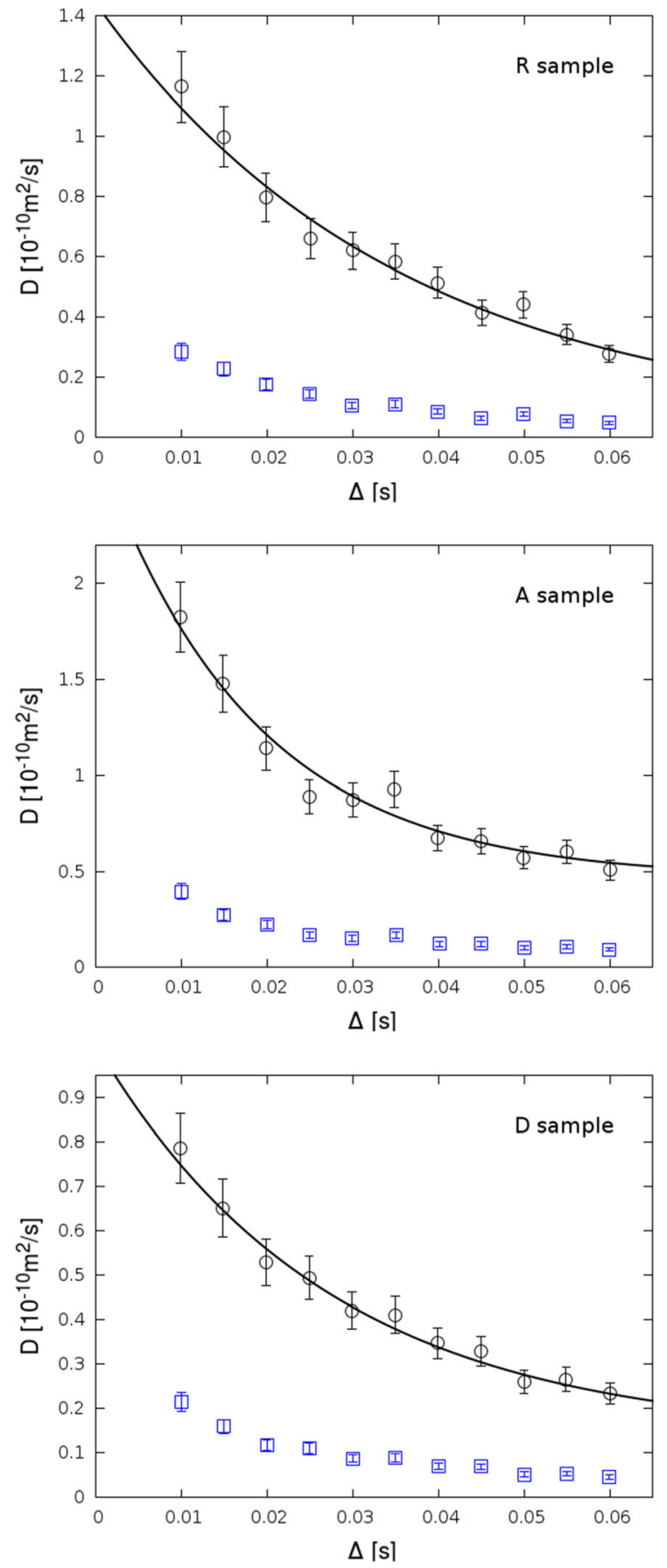

Fig. 1 The phase-1 (black circles) and phase-2 (blue squares) diffusion coefficients as a function of diffusion time $\Delta$ for unaged samples (top: R; middle: A; bottom: D). Artificially aged systems show a similar behavior (data not shown)

expressed through the general relation $r^{2}=6 D_{\alpha} \Delta^{\alpha}$, where $D_{\alpha}$ is either the ordinary self-diffusion $(\alpha=1)$ or the anomalous self-diffusion coefficient $(\alpha \neq 1)$
(Kimmich 1997). In the latter case, $D_{\alpha}$ is measured in $\mathrm{m}^{2} / \mathrm{s}^{\alpha}$. In other terms, the trend of MSD vs. $\Delta$ allows to assess whether the diffusion is anomalous or Fickian, to estimate the correct apparent diffusion coefficient, and also to retrieve direct information on the confinement scale of water at diffusion times close to zero, i.e., $\Delta \rightarrow 0$ (Conti et al. 2017). When MSDs are used, the PFG-STE signal related to two phases in the framework of the Kärger model is still a sum of two simple spin-echo signals:

$$
\begin{aligned}
\frac{E(q, \Delta)}{E(0, \Delta)}= & p_{1} \exp \left[-\frac{1}{6} q^{2} r^{2}(\Delta)_{1}\right] \\
& +p_{2} \exp \left[-\frac{1}{6} q^{2} r^{2}(\Delta)_{2}\right]
\end{aligned}
$$

where $\mathrm{p}_{1}$ and $\mathrm{p}_{2}\left(\mathrm{p}_{1}+\mathrm{p}_{2}=1\right)$ are the water-population fractions of phase-1 and phase-2, respectively. The MSDs of phase-1 as a function of increasing diffusion times in the investigated samples are reported in Fig. 2.

The MSD data reported in Fig. 2 were well fitted by the function $r^{2}(\Delta)_{1}=6 D_{1} \Delta+c_{1}$; therefore, a Fickian diffusion has been assumed over the investigated diffusion-time interval. The apparent diffusion coefficients $D_{1}$, obtained from data shown in Fig. 2, are reported in Fig. 3 and in Table 1.

In addition to that, using the coefficient $\mathrm{c}_{1}$ obtained by the above reported fit, the size of the confining environment of water in the limit $\Delta \rightarrow 0\left(\mathrm{~d}_{1}\right)$ was calculated with the following equation:

$d_{1}=\sqrt{c_{1}}$

After the acidification treatment, the apparent diffusion coefficient $\left(D_{1}\right)$ and the confining size $\left(d_{1}\right)$ of sample $A$ are higher than those of the reference system R (see Figs. 3, 4 and Table 1). This can be ascribed to the fact that acids break cellulose chains in ADs, leading to changes in the conformation and density of the structure (Calvini 2005; Calvini et al. 2008; Stephens et al. 2008). The acidification treatment performed on the samples results in a $\mathrm{S}^{*}$, that is the number of scissions per initial cellulose chain, of about 0.7 , confirming that the hydrolysis of glycosidic bonds took place upon acidification. Hydrolysis results in the opening of the structure, with a significant increase in the pore sizes of ADs located in elementary fibrils at fiber surfaces, which are accessible to acids, as already 

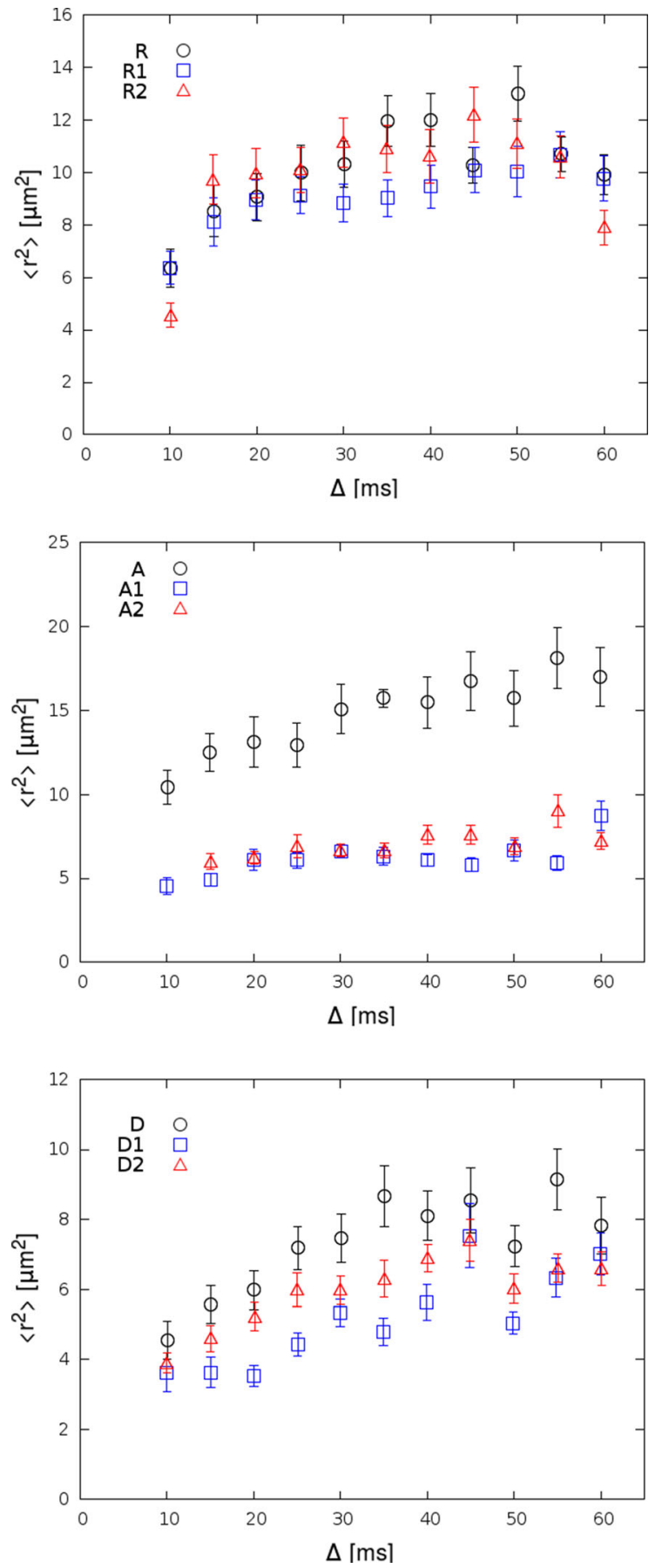

Fig. 2 The MSDs of phase-1 of the investigated samples, at different diffusion times $\Delta$ (top: $\mathrm{R}$ series; middle: A series; bottom: D series)

shown elsewhere (Conti et al. 2014, 2017). The increase in $D_{1}$ and $d_{1}$ following the acidification can also promote the penetration of pollutants inside

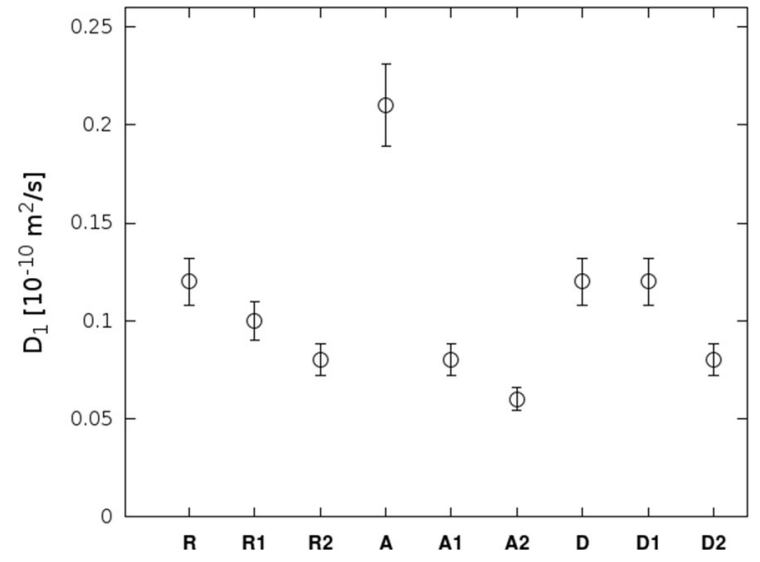

Fig. 3 The apparent diffusion coefficients $\left(D_{1}\right)$ of the investigated samples

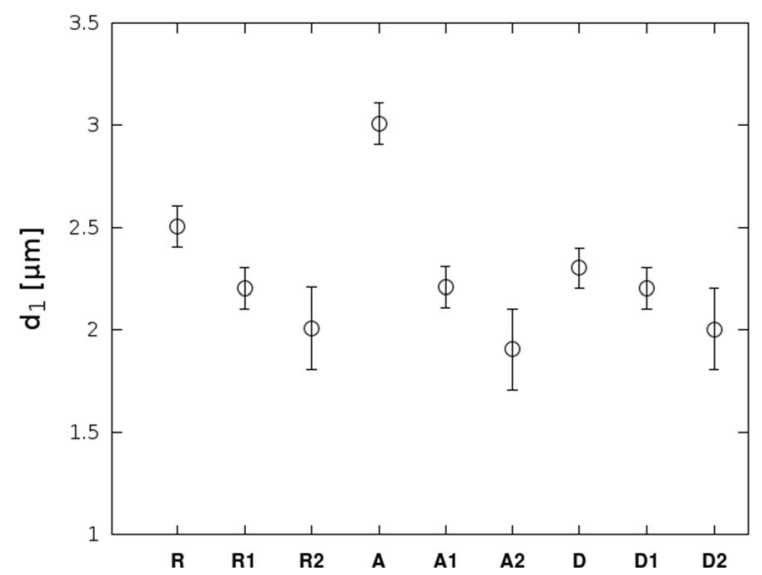

Fig. 4 The confining size for water $\left(d_{1}\right)$ of the investigated samples

paper, which may result in new degradation mechanisms, or enhance the acidification process itself (Menart et al. 2011, 2014).

Interestingly, the neutralization of acids, due to the application of calcium hydroxide nanoparticles, leads to a significant decrease of the apparent diffusion coefficient $\left(D_{1}\right)$ and confining size for water $\left(d_{1}\right)$, as clearly shown in Figs. 3, 4. The neutralization of sulfuric acid probably favors a rearrangement of the smaller fragment of cellulose fibers (Conti et al. 2014). The rearrangement can also be ascribed to the presence of calcium ions: acidity is often interconnected with oxidation in promoting degradation of cellulose (Calvini et al. 2008; Baty et al. 2010). As a result of the oxidation, hydroxyl groups can be oxidized to carboxyl moieties, which are weakly 
acidic. Divalent calcium ions can interact with these groups, which can be located on the same chain or on different cellulose chains, favoring a rearrangement in the cellulosic network (Bukovský 2000; Baglioni et al. 2012; Poggi et al. 2014). The overall effect is a more tightly packed network, as confirmed by the changes in $\mathrm{D}_{1}$ and $\mathrm{d}_{1}$.

In other terms, even if the DP of the deacidified sample is similar to that of the acidic sample, the rearrangement following a deacidification treatment triggers a conformation of the cellulose network that is similar to that of the reference system. It has been already demonstrated that alkaline nanoparticles have a fundamental role in hampering the depolymerization of cellulose, because of the neutralization of acidity (Giorgi et al. 2002; Poggi et al. 2010, 2014; Bastone et al. 2017; Poggi et al. 2017). Here, NMR selfdiffusion measurements are showing that nanoparticles create a tightly packed arrangement even in strongly depolymerized systems, potentially preventing the penetration of pollutants from the outside, and granting a better resistance of the treated paper to further aging. In other terms, the beneficial effects of a deacidification treatment are evident even from the standpoint of cellulose arrangement.

Further support to this scenario is offered by the analysis of the tortuosity parameter $T$. In simple terms, tortuosity is the ratio between the length of a curved path connecting two points and the corresponding straight line between the same two points; therefore, by definition, $T \geq 1$. Even though several models have been developed (Matyka et al. 2008), a general relation between porosity and tortuosity does not exist, apart from a qualitative reduction in tortuosity when porosity increases. Using diffusion coefficients, tortuosity can be expresses by the relation (Callaghan 2011):

$\frac{1}{T}=\frac{D_{1}}{D_{0}}$

where $D_{O}$ is the diffusion coefficient for $\Delta \rightarrow 0$. In our case, $D_{O}$ is experimentally unattainable, and a possible reliable approximation consists in exploiting the diffusion coefficient at the shortest $\Delta(\Delta=10 \mathrm{~ms})$. Under this approximation, the changes in tortuosity with respect to untreated samples, i.e., the relative tortuosity values, have been calculated (see Table 1 and Fig. 5).

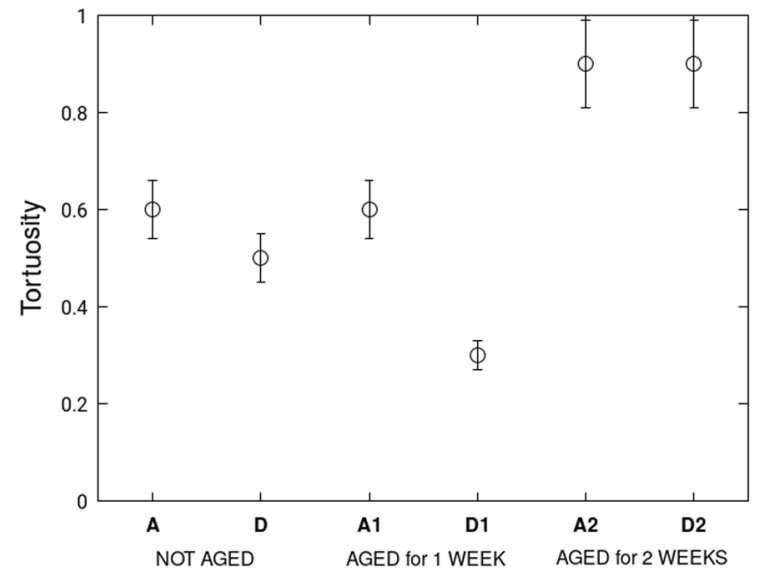

Fig. 5 Relative tortuosity $(T)$ of the investigated samples

The relative tortuosities of $\mathrm{D}$ and $\mathrm{A}$ samples are very similar to each other and are both lower than one. This is probably due to the significant decrease in the degree of polymerization triggered by the acidification process, which makes the obtained network more interconnected. However, even though it looks like the D sample has a porosity comparable to that of the A homologue, the $d_{1}$ and $D_{1}$ data clearly show that the cellulose network in the $\mathrm{D}$ sample retains narrower meshes than the A sample. Because the deacidification process cannot create new glycosidic bonds, the difference among the samples can be only ascribed to a rearrangement in the cellulosic network due to a change in its interconnectivity configuration. Once again, NMR self-diffusion dynamics of water confirms that the deacidification treatment with alkaline nanoparticles re-establishes the pristine network configuration even after a strong acidifying bath, which triggers a pronounced depolymerization of cellulose.

One week of artificial aging under strong conditions promoted further degradation in the polymeric network. As a result, sample A1 displays a significant decrease in the apparent diffusion coefficient $\left(D_{1}\right)$ and confining size for water $\left(\mathrm{d}_{1}\right)$. The environmental conditions of the artificial aging, i.e., high temperature and relative humidity, promote the mobility of cellulose fragments and the cleavage of cellulose chains at random sites, which allows for a more compact arrangement of the fragmented cellulose molecules (Zervos 2007). Further depolymerization of cellulose following artificial aging is confirmed by $\mathrm{S}^{*}$ values, which increase from 0.7 to 1.6, as reported in Table 1. 
Sample D1 shows an apparent diffusion coefficient higher than those of $\mathrm{A} 1$ and $\mathrm{R} 1$, even though to a different extent; conversely, water confining sizes remain similar. This is likely due to the significant slowing down of the hydrothermal aging caused by the buffering action of nanoparticles. In fact, less fragments of cellulose, which are those capable of undergoing rearrangement, are available when hydrolysis is hindered. Therefore, no or slight changes in $\mathrm{D}_{1}$ and $\mathrm{d}_{1}$ occur in the deacidified sample. These data are corroborated by the $\mathrm{S}^{*}$ values reported in Table 1 . Overall, the positive effect of the deacidification treatment on cellulose arrangement is retained even after one week of artificial aging.

After 2 weeks, the parameters characterizing the deacidified samples become very similar to those associated to the reference and acidic samples (Figs. 3, 4, respectively). This suggests that, for what concerns the self-diffusion dynamics of water, a common state is reached in cellulose arrangement, and this state is achieved independently of the sample treatment history. However, after the second week of aging, the DP of R2 significantly decreases, while neither A2 nor D2 shows significant changes in $\mathrm{S}^{*}$ (see Table 1). It has been shown that there is a relation between hydrothermal aging mechanisms, such as chain scission and crosslinking, and hornification, which causes structural changes in cellulose due to irreversible intra-fiber hydrogen bonding (Kato and Cameron 1999). It is worth noting that, except for chain cleavage, none of the remaining mechanisms affects the DP of cellulose. We can therefore conclude that, after 2 weeks at high temperature and relative humidity, the mechanisms that do not affect the DP, such as irreversible intra-fiber hydrogen bonding, are more effective in changing the texture of cellulose fibers than those concerning chain scission, especially for samples $\mathrm{A}$ and $\mathrm{D}$, which underwent a strong acidification treatment before aging. Nevertheless, alkaline nanoparticles are proven fundamental in hampering the degradation of acidic paper, as shown by the significantly lower $S^{*}$ values obtained for D2 sample with respect to A2 sample (see Table 1).

Relative tortuosities after 1 and 2 weeks of artificial aging are reported in Table 1 and Fig. 5. D1 shows a lower tortuosity than A1, which is consistent with the corresponding apparent diffusion coefficients reported in Fig. 3. The protection from aging due to the presence of alkaline nanoparticles makes D1 sample more similar to A than to A1. It is worth noting that, after one week of aging, $T$ values are significantly lower than 1 . This confirms that, for short-term aging, depolymerization is still the dominant factor in driving cellulose network configuration. At longer aging times, as indicated above, a common state is reached, independently of the sample treatment history, likely due to the prominence of hornification over depolymerization.

\section{Conclusions}

The changes in the cellulosic network following an acidification bath and a consequent deacidification treatment have been indirectly investigated by NMR self-diffusion dynamics of water, and related to changes in the degree of polymerization in response to artificial aging.

The acidification treatment results in the opening of the structure, with a significant increase in pore sizes of ADs located in elementary fibrils at fiber surfaces. This may promote the penetration of air pollutants, with possible detrimental effect to paper conservation. The neutralization of acids due to alkaline nanoparticles leads to a rearrangement of cellulose fibers into a more tightly packed conformation, resembling the cellulosic network of the reference system.

After 1 week of aging, the acidic sample displays a more compact arrangement of the fragmented molecules, due to a further cellulose depolymerization, as confirmed by DP measurements. Correspondingly, the hampering of the hydrothermal aging due to nanoparticles results in an apparent diffusion coefficient higher than those of $\mathrm{A} 1$ and $\mathrm{R} 1$, confirming the beneficial effect of the applied deacidification treatment even upon aging.

After 2 weeks, a competition between hydrothermal aging mechanisms, such as chain scission and crosslinking, and hornification, which causes irreversible intra-fiber hydrogen bonding, probably occurs in the investigated systems. For samples A and D, which underwent a strong acidification treatment before aging, irreversible hydrogen bonding has a more pronounced effect on the texture of cellulose fibers than chain scission, leading to a decrease in the water diffusive parameters without significant changes in DP. However, the DP of the deacidified sample is close to that of the reference system, further 
corroborating the idea that calcium hydroxide nanoparticles are an effective tool to hamper the degradation of cellulose induced by acids and aging under strong environmental conditions, even from the point of view of cellulose network arrangement.

Acknowledgments This work was partly supported by CSGI and the European Union's Horizon 2020 research and innovation programme under grant agreement No 646063 [Nanorestart Project].

\section{Compliance with ethical standards}

Conflicts of interest There are no conflicts to declare.

\section{References}

Afsharpour M, Hadadi M (2014) Titanium dioxide thin film: environmental control for preservation of paper-art-works. J Cult Herit 15:569-574. https://doi.org/10.1016/j.culher. 2013.10.008

Arroyo OH, Jalbert J, Fofana I, Ryadi M (2017) Temperature dependence of methanol and the tensile strength of insulation paper: kinetics of the changes of mechanical properties during ageing. Cellulose 24:1031-1039. https://doi. org/10.1007/s10570-016-1123-7

Baglioni P, Chelazzi D, Giorgi R, Poggi G (2012) Nanoparticles for the conservation of cultural heritage: paper and wood. In: Somasundaran P (ed) The encyclopedia of surface and colloid science, 2nd edn. Taylor \& Francis, New York, pp $1-16$

Bastone S, Chillura Martino DF, Renda V et al (2017) Alcoholic nanolime dispersion obtained by the insolubilisation-precipitation method and its application for the deacidification of ancient paper. Colloids Surfaces A Physicochem Eng Asp 513:241-249. https://doi.org/10.1016/j.colsurfa.2016. 10.049

Baty JW, Maitland CL, Minter W et al (2010) Deacidification for the conservation and preservation of paper-based works: a review. BioResources 5:1955-2023

Bukovský V (2000) The influence of light on ageing of newsprint paper. Restaurator 21:55-76. https://doi.org/10.1515/ REST.2000.55

Callaghan PT (2011) Translational dynamics and magnetic resonance: principles of pulsed gradient spin echo NMR. Oxford University Press, New York

Calvini P (2005) The influence of levelling-off degree of polymerisation on the kinetics of cellulose degradation. Cellulose 12:445-447. https://doi.org/10.1007/s10570-0052206-Z

Calvini P, Gorassini A, Merlani AL (2008) On the kinetics of cellulose degradation: looking beyond the pseudo zero order rate equation. Cellulose 15:193-203. https://doi.org/ 10.1007/s10570-007-9162-8

Conti A, Poggi G, Baglioni P et al (2014) On the macromolecular cellulosic network of paper: changes induced by acid hydrolysis studied by NMR diffusometry and relaxometry. Phys Chem Chem Phys 16:8409-8417. https://doi.org/10.1039/c4cp00377b

Conti A, Palombo M, Parmentier A et al (2017) Two-phase water model in the cellulose network of paper. Cellulose 24:3479-3487. https://doi.org/10.1007/s10570-017-1338-2

Ding S-Y, Zhao S, Zeng Y (2014) Size, shape, and arrangement of native cellulose fibrils in maize cell walls. Cellulose 21:863-871. https://doi.org/10.1007/s10570-013-0147-5

Dobrodskaya TV, Egoyants PA, Ikonnikov VK et al (2004) Treatment of paper with basic agents in alcohols and supercritical carbon dioxide to neutralize acid and prolong storage time. Russ J Appl Chem 77:2017-2021. https://doi. org/10.1007/s11167-005-0211-5

Donaldson L (2007) Cellulose microfibril aggregates and their size variation with cell wall type. Wood Sci Technol 41:443-460. https://doi.org/10.1007/s00226-006-0121-6

Dupont A-L, Mortha G (2004) Comparative evaluation of sizeexclusion chromatography and viscometry for the characterisation of cellulose. J Chromatogr A 1026:129-141. https://doi.org/10.1016/j.chroma.2003.11.002

Fengel D, Wegener G (1984) Wood: chemistry, ultrastructure, reactions. Walter De Gruyter, Berlin and New York

Fernandes AN, Thomas LH, Altaner CM et al (2011) Nanostructure of cellulose microfibrils in spruce wood. Proc Natl Acad Sci 108:E1195-E1203. https://doi.org/10.1073/pnas. 1108942108

Giorgi R, Dei L, Ceccato M et al (2002) Nanotechnologies for conservation of cultural heritage: paper and canvas deacidification. Langmuir 18:8198-8203. https://doi.org/ 10.1021/la025964d

Giorgi R, Bozzi C, Dei L et al (2005a) Nanoparticles of $\mathrm{Mg}(\mathrm{OH})_{2}$ : synthesis and application to paper conservation. Langmuir 21:8495-8501. https://doi.org/10.1021/ la050564m

Giorgi R, Chelazzi D, Baglioni P (2005b) Nanoparticles of calcium hydroxide for wood conservation. The deacidification of the Vasa Warship. Langmuir 21:10743-10748. https://doi.org/10.1021/la0506731

Kärger J, Pfeifer H, Heink W (1988) Principles and application of self-diffusion measurements by nuclear magnetic resonance. Adv Magn Opt Reson 12:1-89. https://doi.org/10. 1016/B978-0-12-025512-2.50004-X

Kato KL, Cameron RE (1999) A review of the relationship between thermally-accelerated ageing of paper and hornification. Cellulose 6(23):40

Kimmich R (1997) NMR-tomography, diffusometry, relaxometry. Springer, Berlin. https://doi.org/10.1007/978-3642-60582-6

Kulasinski K, Guyer R, Derome D, Carmeliet J (2015) Water diffusion in amorphous hydrophilic systems: a stop and go process. Langmuir 31:10843-10849. https://doi.org/10. 1021/acs.langmuir.5b03122

Matyka M, Khalili A, Koza Z (2008) Tortuosity-porosity relation in porous media flow. Phys Rev E 78:026306. https:// doi.org/10.1103/PhysRevE.78.026306

Menart E, De Bruin G, Strlič M (2011) Dose-response functions for historic paper. Polym Degrad Stab 96:2029-2039. https://doi.org/10.1016/j.polymdegradstab.2011.09.002

Menart E, de Bruin G, Strlič M (2014) Effects of NO2 and acetic acid on the stability of historic paper. Cellulose 21:3701-3713. https://doi.org/10.1007/s10570-014-0374-4 
Müller M, Riekel C, Vuong R, Chanzy H (2000) Skin/core micro-structure in viscose rayon fibres analysed by X-ray microbeam and electron diffraction mapping. Polymer (Guildf) 41:2627-2632. https://doi.org/10.1016/S00323861(99)00433-4

Niskanen K (1998) Paper physics. Fapet Oy, Helsinky (FIN)

Paajanen A, Ceccherini S, Maloney T, Ketoja JA (2019) Chirality and bound water in the hierarchical cellulose structure. Cellulose 26:5877-5892. https://doi.org/10.1007/ s10570-019-02525-7

Palladino N, Hacke M, Poggi G et al (2020) Nanomaterials for combined stabilisation and deacidification of cellulosic materials - the case of iron-tannate dyed cotton. Nanomaterials 10:900. https://doi.org/10.3390/nano10050900

Poggi G, Giorgi R, Toccafondi N et al (2010) Hydroxide nanoparticles for deacidification and concomitant inhibition of iron-gall ink corrosion of paper. Langmuir 26:19084-19090. https://doi.org/10.1021/la1030944

Poggi G, Toccafondi N, Melita LN et al (2014) Calcium hydroxide nanoparticles for the conservation of cultural heritage: new formulations for the deacidification of cellulose-based artifacts. Appl Phys A 114:685-693. https:// doi.org/10.1007/s00339-013-8172-7

Poggi G, Sistach MC, Marin E et al (2016a) Calcium hydroxide nanoparticles in hydroalcoholic gelatin solutions (GeolNan) for the deacidification and strengthening of papers containing iron gall ink. J Cult Herit 18:250-257. https:// doi.org/10.1016/j.culher.2015.10.005

Poggi G, Toccafondi N, Chelazzi D et al (2016b) Calcium hydroxide nanoparticles from solvothermal reaction for the deacidification of degraded waterlogged wood. J Colloid Interface Sci 473:1-8. https://doi.org/10.1016/j.jcis.2016. 03.038

Poggi G, Giorgi R, Mirabile A et al (2017) A stabilizer-free nonpolar dispersion for the deacidification of contemporary art on paper. J Cult Herit 26:44-52. https://doi.org/10.1016/j. culher.2017.02.006

Schuster KC, Aldred P, Villa M et al (2003) Characterising the emerging Lyocell fibres structures by ultra small angle neutron scattering (USANS). Lenzinger Berichte 82:107-117

Sequeira S, Casanova C, Cabrita E (2006) Deacidification of paper using dispersions of $\mathrm{Ca}(\mathrm{OH}) 2$ nanoparticles in isopropanol. Study of efficiency. J Cult Herit 7:264-272. https://doi.org/10.1016/j.culher.2006.04.004

Stefanis E, Panayiotou C (2007) Protection of lignocellulosic and cellulosic paper by deacidification with dispersions of micro- and nano-particles of $\mathrm{Ca}(\mathrm{OH})_{2}$ and $\mathrm{Mg}(\mathrm{OH})_{2}$ in alcohols. Restaurator 28:185-200. https://doi.org/10.1515/ REST.2007.185

Stefanis E, Panayiotou C (2008) Study of the photochemical stability of paper deacidified with dispersions of $\mathrm{Ca}(\mathrm{OH})_{2}$ and $\mathrm{Mg}(\mathrm{OH})_{2}$ nanoparticles in alcohols. Restaurator 29:125-138. https://doi.org/10.1515/rest.2008.007

Stephens CH, Whitmore PM, Morris HR, Bier ME (2008) Hydrolysis of the amorphous cellulose in cotton-based paper. Biomacromol 9:1093-1099. https://doi.org/10. 1021/bm800049w

Strlič M, Kolar J (2005) Ageing and stabilization of paper. National and University Library, Ljubljana

Topgaard D, Söderman O (2001) Diffusion of water absorbed in cellulose fibers studied with 1H-NMR. Langmuir 17:2694-2702. https://doi.org/10.1021/la0009821

UNI 8282 (1994) Cellulose in dilute solutions-determination of limiting viscosity number-method in cupri-ethylenediamine (CED) solution-equivalent to the ISO standard $5351 / 1$

Wouters J (2008) Coming soon to a library near you? Science 322:1196-1198

Xu Q, Poggi G, Resta C et al (2020) Grafted nanocellulose and alkaline nanoparticles for the strengthening and deacidification of cellulosic artworks. J Colloid Interface Sci 576:147-157. https://doi.org/10.1016/j.jcis.2020.05.018

Yanjuan W, Yanxiong F, Wei T, Chunying L (2013) Preservation of aged paper using borax in alcohols and the supercritical carbon dioxide system. J Cult Herit 14:16-22. https://doi.org/10.1016/j.culher.2012.02.010

Zervos S (2007) Characterization of changes induced by ageing to the microstructure of pure cellulose paper by use of a gravimetric water vapour adsorption technique. Cellulose 14:375-384. https://doi.org/10.1007/s10570-007-9125-0

Zervos S, Alexopoulou I (2015) Paper conservation methods: a literature review. Cellulose 22:2859-2897. https://doi.org/ 10.1007/s10570-015-0699-7

Zervos S, Moropoulou A (2005) Cotton cellulose ageing in sealed vessels. Kinetic model of autocatalytic depolymerization. Cellulose 12:485-496. https://doi.org/10.1007/ s10570-005-7131-7

Zhao H, Kwak JH, Conrad Zhang Z et al (2007) Studying cellulose fiber structure by SEM, XRD, NMR and acid hydrolysis. Carbohydr Polym 68:235-241. https://doi.org/ 10.1016/j.carbpol.2006.12.013

Publisher's Note Springer Nature remains neutral with regard to jurisdictional claims in published maps and institutional affiliations. 